\title{
MOUVEMENTS PRESQUE-PÉRIODIQUES D'UNE CORDE VIBRANTE EN PRÉSENCE D'UN OBSTACLE FIXE, RECTILIGNE OU PONCTUEL
}

\author{
Henri Cabannes $\nmid$ el alain Hakaux $\neq$ \\ Université Pierre et Marie Curie, 4 place Jussieu, 75005 Paris, France
}

\begin{abstract}
We consider the plane motions of a vibrating string, fixed at both ends, in the presence of a straight fixed obstacle, parallel to the equilibrium position of the string. The rebound of the string on the obstacle obeys to the laws of perfect reflection. We prove that, under rather general conditions, the motion of the string initially at rest, is an almost periodic function of the time. When a certain ratio is rational, the motion is periodic and we compute the period; then we prove the existence of almost periodic but non-periodic motions. In a last part we consider the case of a fixed mass-point obstacle placed on the normal mid-line of the segment joining the fixed ends of the string; we obtain similar results.
\end{abstract}

\section{INTRODUCTION}

Les premières recherches relatives aux mouvements des cordes vibrantes remontent à d'Alembert et furent publiées en 1761 [1]. L'importance des mouvements vibratoires dans tous les chapitres de la Mécanique est à l'origine du nombre considérable de travaux consacrés au problème des cordes vibrantes. Cependant l'étude du mouvement d'une corde qui vibre en présence d'un obstacle a été entreprise pour la première fois seulement en 1975, par Amerio et Prouse [2]. Ces auteurs ont démontré l'existence de la solution lorsque la corde, fixée à ses deux extrémités, oscille en présence d'un obstacle fixe, rectiligne, parallèle à la position d'équilibre; ce résultat a été étendu par Schatzman au cas des obstacles concaves [3]. Le cas des obstacles ponctuels a été envisagé par Reder [4] et Cabannes [5]. Il y a lieu de citer également les travaux de Betro et Gotusso [6] ainsi que ceux de Citrini [7].

Dans le travail qui suit, nous reprenons d'abord le cas d'un obstacle rectiligne envisagé par Amerio et Prouse et nous nous intéressons, non pas à l'existence du mouvement, mais à sa nature. Nous démontrons que, lorsque la corde est initialement au repos dans une position suffisamment régulière, c'est-à-dire telle que l'élongation, distance à la position d'équilibre, considérée comme une fonction de l'abcisse curviligne, soit non décroissante sur une partie de la corde et non croissante sur l'autre partie, alors le mouvement est une fonction presque-périodique du temps. Les cas particuliers où cette fonction est périodique sont précisés. Cette étude fait suite à la publication [8] dans laquelle seuls des mouvements périodiques étaient considérés, et à la publication [9] dans laquelle la position initiale de la corde présentait un axe de symétrie. Dans le dernier paragraphe nous envisageons le cas d'un obstacle ponctuel placé sur la médiatrice du segment qui joint les extrémités de la corde. A l'instant initial, la corde est au repos dans une position symétrique par rapport à la médiatrice précédente et satisfaisant les hypothèses déjà précisées. Le mouvement est encore une fonction presque-périodique du temps, fonction qui est périodique dans certains cas.

\section{ENONCE DU PROBLÈME}

Utilisant des variables sans dimensions, nous nous plaçons dans un plan rapporté

+Laboratoire de Méchanique Théorique, associé au C.N.R.S.

‡Laboratoire d'Analyse Numérique et Fonctionelle, associé au C.N.R.S. 
au repère orthonormé $O x u$. et nous considérons les petites oscillations d'une corde vibrante dont les extrémités $\left(x= \pm \frac{1}{2}, u=0\right)$ sont fixes. Les oscillations libres de la corde sont perturbées par la présence d'un obstacle fixe: $u=-h$, contre lequel elle peut rebondir suivant les lois de la réflexion parfaite; la vitesse du point de la corde qui heurte l'obstacle est multipliée par -1 au cours du choc.

A l'instant initial (Fig. 1), la corde est au repos dans une position $u(x, 0)=u_{0}(x)$ qui vérifie les hypothèses suivantes

$$
\left.\begin{array}{l}
u_{0}(x) \text { est continue pour }-\frac{1}{2} \leqslant x \leqslant \frac{1}{2} \\
\mathrm{~d} u_{0} / \mathrm{d} x \geqslant 0 \text { pour }-\frac{1}{2} \leqslant x \leqslant a<\frac{1}{2} \\
\mathrm{~d} u_{0} / \mathrm{d} x \leqslant 0 \text { pour }-\frac{1}{2}<a \leqslant x \leqslant \frac{1}{2} \\
u_{0}\left( \pm \frac{1}{2}\right)=0 \text { ct } u_{0}(a)=1 \\
u_{0}(x)>-h \text { pour }-\frac{1}{2} \leqslant x \leqslant \frac{1}{2} .
\end{array}\right\}
$$

Aussi longtemps que la corde n'a pas rencontré l'obstacle, le mouvement de la corde est l'oscillation libre $u=w(x, t)$ solution de l'équation des ondes, équation (1), satisfaisant les conditions initiales (2) et les conditions aux limites (3)

$$
\begin{gathered}
\square u \equiv \frac{\hat{\partial}^{2} u}{\partial t^{2}}-\frac{\partial^{2} u}{\partial x^{2}}=0 \\
u(x, 0)=u_{0}(x), \quad(\hat{c} u / \partial t)(x, 0)=0 \\
u\left( \pm \frac{1}{2}, t\right)=0 \text { pour } t \geqslant 0 .
\end{gathered}
$$

Pour déterminer le domaine de validité de la solution $u=w(x, t)$, Amerio et Prouse [2] considèrent le domaine $E$ du plan $x-t$, dans lequel on a $w(x, t)<-h$, ainsi que les caractéristiques (droites $x \pm t=$ constante) tangentes à la frontière de ce domaine (Fig. 2). La ligne $A^{\prime} A B B^{\prime}$ ainsi formée, d'équation $t=\tau(x)$, comprend des portions caractéristiques telles que $A^{\prime} A$ et $B B^{\prime}$ sur lesquelles on a $\mathrm{d} \tau / \mathrm{d} x= \pm 1$ et des portions non caractéristiques telles que $A B$ sur lesquelles on a $|\mathrm{d} \tau / \mathrm{d} x|<1$; cette ligne est appelée ligne d'influence car, au-delà, le mouvement de la corde est influencé par la présence de l'obstacle.

La détermination du mouvement au-delà de la première ligne d'influence est une suite de problèmes de Cauchy et de Goursat. Sur la Fig. 2 sont tracées les caractéristiques issues des points $A, B, A^{\prime}$ et $B^{\prime}$; ces caractéristiques déterminent les régions (2)-(5). Dans la région (2), le mouvement est déterminé par les données sur l'arc $A B$ de la fonction $u(x ; t)=-h$ et de la dérivée $(\partial u / \partial t)=-(\hat{c} w / \hat{c} t)$. On en déduit

$$
u_{2}(x, t)=-2 h-w(x, t)
$$

Introduisant les coordonnées caractéristiques $\xi=x+t, \eta=x-t$ et posant $\hat{u}(\xi, \eta)=u(x, t)$,

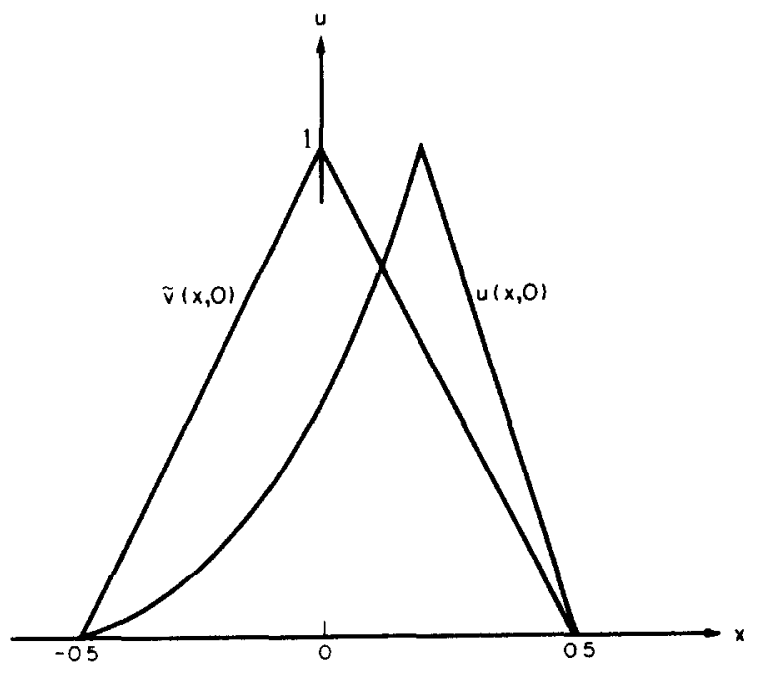

Fig 1 Données initiales 


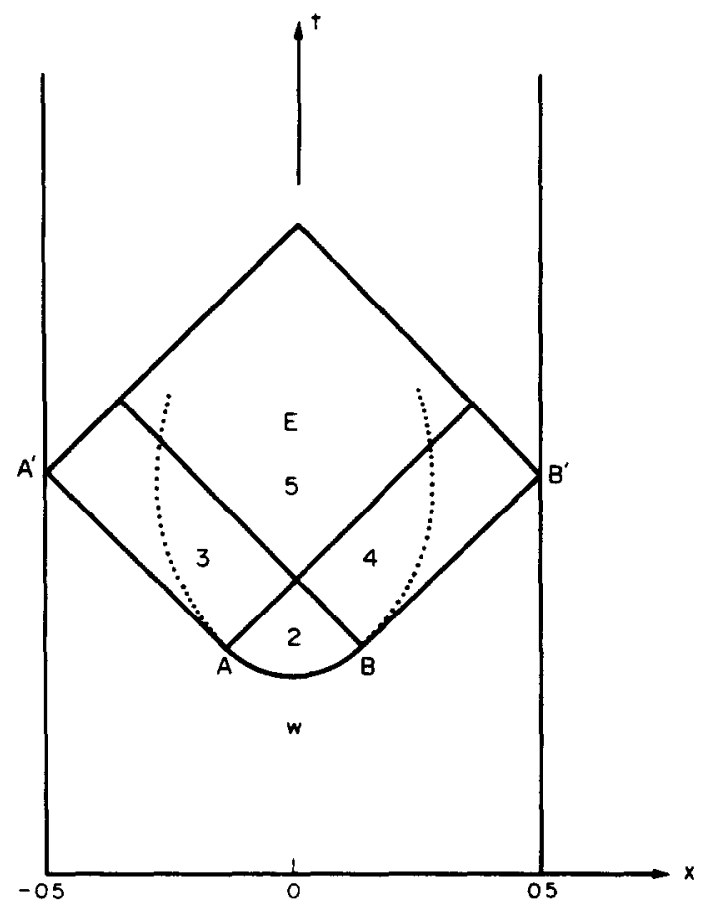

Fig 2. Ligne d'influence

on obtient successivement

$$
\begin{aligned}
\hat{u}_{3}(\xi, \eta) & =\hat{w}\left(\xi_{A}, \eta\right)+\hat{u}_{2}\left(\xi, \eta_{A}\right)-\hat{u}\left(\xi_{A}, \eta_{A}\right) \\
& =\hat{w}\left(\xi_{A}, \eta\right)-\hat{w}\left(\xi, \eta_{A}\right)-h \\
\hat{u}_{4}(\xi, \eta) & =\hat{w}\left(\xi, \eta_{B}\right)+\hat{u}_{2}\left(\xi_{B}, \eta\right)-\hat{u}\left(\xi_{B}, \eta_{B}\right) \\
& =\hat{w}\left(\xi, \eta_{B}\right)-\hat{w}\left(\xi_{B}, \eta\right)-h \\
\hat{u}_{5}(\xi, \eta) & =\hat{u}_{3}\left(\xi_{B}, \eta\right)+\hat{u}_{4}\left(\xi, \eta_{A}\right)-\hat{u}\left(\xi_{B}, \eta_{A}\right) \\
& =\hat{w}\left(\xi, \eta_{B}\right)+\hat{w}\left(\xi_{A}, \eta\right)-\hat{w}\left(\xi_{B}, \eta_{A}\right) .
\end{aligned}
$$

On retrouve ainsi, après le premier contact, une nouvelle oscillation libre qui est valable jusqu'à la seconde ligne d'influence.

\section{CHANGEMENT DE VARIABLES}

La méthode que nous venons d'indiquer permet d'étudier le mouvement de la corde pour toutes les valeurs positives du temps et également pour toutes les valeurs négatives. Elle ne permet pas cependant d'établir les propriétés de presque-périodicité que nous souhaitons démontrer. Pour établir ces propriétés, nous utiliserons la méthode dite du changement de variables. Désignant par $F$ une fonction pour le moment arbitraire, nous faisons correspondre à toute fonction $v(x, t)$ la nouvelle fonction $u(x, t)$ définie par la formule

$$
u(x, t)=v\left\{\frac{F(x+t)+F(x-t)}{2}, \frac{F(x+t)-F(x-t)}{2}\right\} .
$$

On obtient, lorsque la fonction $F$ est deux fois dérivable et en posant $2 X=F(x+t)+F(x-t)$ et $2 T=F(x+t)-F(x-t)$

$$
\left.\begin{array}{l}
\frac{\hat{\partial} u}{\partial x}=\frac{\hat{\partial} v}{\partial X} \frac{F^{\prime}(x+t)+F^{\prime}(x-t)}{2}+\frac{\partial v}{\partial T} \frac{F^{\prime}(x+t)-F^{\prime}(x-t)}{2} \\
\frac{\hat{c} u}{\hat{\partial} t}=\frac{\hat{\partial} v}{\partial X} \frac{F^{\prime}(x+t)-F^{\prime}(x-t)}{2}+\frac{\partial v}{\partial T} \frac{F^{\prime}(x+t)+F^{\prime}(x-t)}{2}
\end{array}\right\}
$$

$\square u(x, t)=F^{\prime}(x+t) F^{\prime}(x-t) \square v(X, T)$. 
Il en résulte que, si la fonction $v(x, t)$ est une solution de l'équation des ondes, il en est de même de la fonction $u(x, t)$. Ainsi, à partir d'une solution de l'équation (1), on obtient par la formule (6) une famille de solutions dépendant de la fonction $F$. Naturellement les conditions initiales sont différentes

$$
\left.\begin{array}{c}
u(x, 0)=v\{F(x), 0\} \\
\left.\frac{\partial u}{\partial t}(x, 0)=\frac{\partial v}{\partial t}\{F(x), 0\} F^{\prime}(x) .\right\}
\end{array}\right\}
$$

Lorsque la solution $v(x, t)$ représente le mouvement d'une corde initialement au repos, il en est de même de la solution $u(x, t)$. Il est alors intéressant de chercher $s i$, lorsque la solution $v(x, t)$ vérifie les conditions aux limites (3), il peut en être de même pour la solution $u(x, t)$. Pour qu'il en soit ainsi, il suffit d'imposer à la fonction $F$ les conditions (10) ou les conditions (11) qui sont équivalentes

$$
\begin{aligned}
& \left.\begin{array}{rl}
F\left(-\frac{1}{2}+t\right)+F\left(-\frac{1}{2}-t\right) & =-1 \\
F\left(\frac{1}{2}+t\right)+F\left(\frac{1}{2}-t\right) & =1
\end{array}\right\} \\
& \left.\begin{array}{l}
F(y+1)=1-F(-y) \\
F(y+2)=2+F(y)
\end{array}\right\}
\end{aligned}
$$

La première des relations (11) indique que le graphe de la fonction $F(y)$ admet le point $y=F=\frac{1}{2}$ comme centre de symétrie, tandis que la seconde de ces relations indique que la fonction $F(y)-y$ est périodique de période 2 . Lorsque les conditions initiales sont données sur l'intervalle $-\frac{1}{2} \leqslant x \leqslant \frac{1}{2}$, la première des relations (9) détermine sur cet intervalle la fonction $F$ qui fait correspondre les solutions $u(x, t)$ et $v(x, t)$; la correspondance n'est possible que si les répartitions initiales des vitesses sont liées par la seconde des formules (9), ce qui est le cas, en particulier, lorsque la corde est initialement au repos pour les mouvements correspondant aux solutions $u(x, t)$ et $v(x, t)$. Les formules (11) définissent ensuite la fonction $F$ sur tout l'axe réel; comme $F(y)=F(y)-y+y$ est la somme d'une fonction périodique et de la fonction identique, $F(y)$ est uniformément continue sur tout l'axe réel.

A partir d'une solution de l'équation (1) satisfaisant les conditions aux limites (3), on a obtenu une famille de solutions satisfaisant la même équation et les mêmes conditions aux limites. Il est remarquable qu'il soit en outre possible de choisir la fonction $F$ de façon que, si $v(x, t)$ représente un mouvement de la corde en présence de l'obstacle $u=-h, u(x, t)$ représente aussi un mouvement de la corde en présence du même obstacle. Au moment du contact (arc $A B$ de la ligne d'influence), en écrivant pour plus de clarté $v(X, T)$ à la place

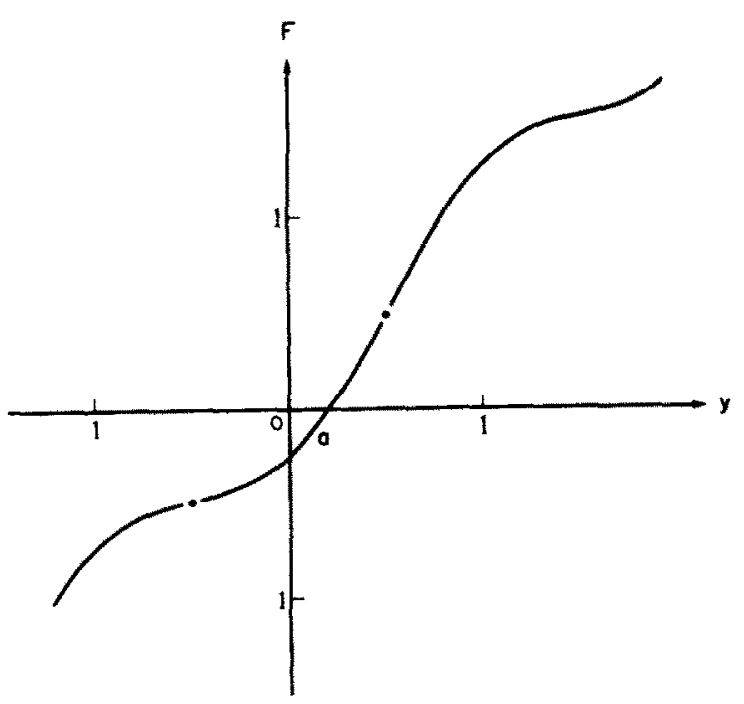

F1g 3 Changement de variable. 
de $v(x, t)$, on a

$$
\left.\begin{array}{cc}
v(X, T)=-h, \quad \frac{\partial v}{\partial T} \leqslant 0 \text { juste avant le contact } & \\
\frac{\partial v}{\partial X} \mathrm{~d} X+\frac{\partial v}{\partial T} \mathrm{~d} T=0 & \} \\
\frac{\partial u}{\partial t}=\frac{\partial v}{\partial T}\left\{\frac{F^{\prime}(x+t)}{2}\left(1-\frac{\mathrm{d} T}{\mathrm{~d} X}\right)+\frac{F^{\prime}(x-t)}{2}\left(1+\frac{\mathrm{d} T}{\mathrm{~d} X}\right)\right\} & \frac{\partial T}{\partial t}=\frac{F^{\prime}(x+t)+F^{\prime}(x-t)}{2}
\end{array}\right\}
$$

Sur la partie non caractéristique de la ligne d'influence, arc $A B$ de la Fig. 2, on a $v=-h$, $|\mathrm{d} T / \mathrm{d} X| \leqslant 1 ;(\partial v / \partial T)$ est négatif ou nul juste avant le contact, et est multiplié par -1 au cours du contact. D'après les formules (6) et (13) on a aussi $u=-h$, et $(\partial u / \partial t)$ est multiplié par -1 au cours du contact; pour satisfaire en outre la condition $(\partial u / \partial t) \leqslant 0$ juste avant le contact, il suffit de choisir pour fonction $F$ une fonction non décroissante, car alors les dérivées $(\partial u / \partial t)$ et $(\partial v / \partial T)$ ont même signe, et, $(\partial T / \partial t)$ étant positif, il y a correspondance entre les instants avant le contact (et les instants après le contact) pour les fonctions $v(X, T)$ et $u(x, t)$. Nous choisissons donc la fonction $F$ non décroissante. Ce choix étant fait, la formule (6) permet d'apporter de grandes simplifications à l'étude du mouvement de la corde en présence de l'obstacle $u=-h$.

\section{MOUVEMENT DE LA COR DE PINCÉE SYMÉTRIQUE}

La solution la plus simple que nous désignerons par $\tilde{v}(x, t)$ correspond au cas de la corde pincée symétrique, c'est-à-dire aux données initiales suivantes

$$
\left.\begin{array}{l}
\tilde{v}(x, 0)=1-2|x| \\
\frac{\partial \bar{v}}{\partial t}(x, 0)=0 .
\end{array}\right\}
$$

On suppose $h$ compris entre 0 et 1 afin que la corde vienne heurter l'obstacle au cours du mouvement. Les valeurs de la fonction $\tilde{v}(x, t)$ sont indiquées sur la Fig. 4. La première

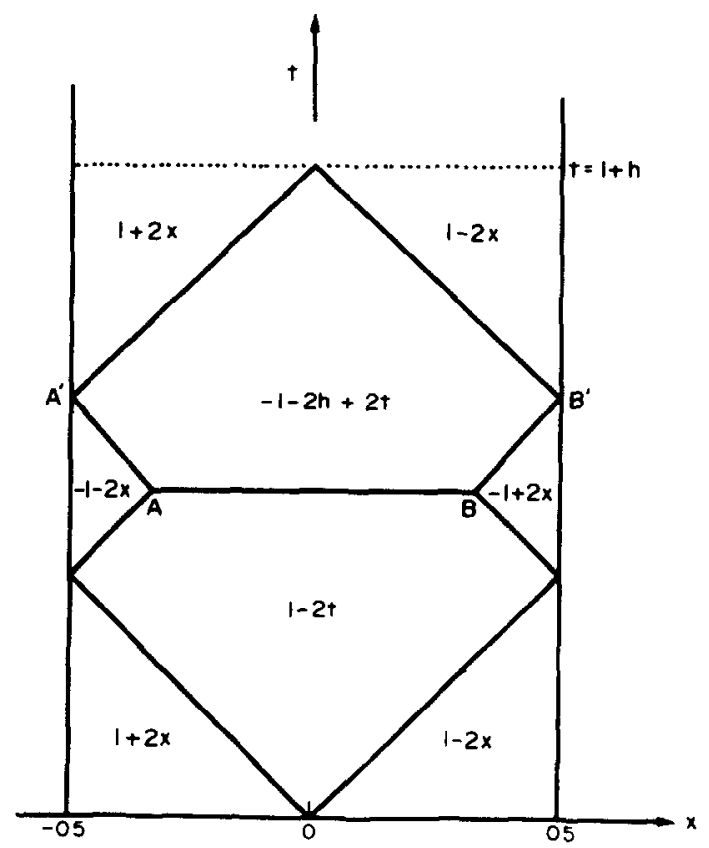

Fig. 4. Mouvement de la corde pincée symétrique; obstacle rectiligne $u=-h$. 
ligne d'influence est constituée par le segment de droite $A B(2 t=1+h, 2|x| \leqslant 1-h)$ et par les caractéristiques $A^{\prime} A$ et $B B^{\prime}$. A l'instant $t=1+h / 2$. la corde rencontre robstacle le long du segment $u=-h,|x| \leqslant 1-h / 2$ avec la vitesse uniforme -2 , vitesse qui passe instantanément de la valeur -2 à la valeur 2 . Le mouvement continue ensuite de façon symétrique

$$
\tilde{v}(x, t)=\tilde{v}(x, 1+h-t) \text { pour } \frac{1+h}{2} \leqslant t \leqslant 1+h .
$$

A l'instant $t=1+h$, on est ramené aux conditions initiales, si bien que le mouvement est périodique de période $1+h$.

\section{ETUDE DU CAS GENERAL}

Nous supposons maintenant que la corde est initialement au repos dans une position satisfaisant les hypothèses $\left(\mathscr{H}^{*}\right)$. Des résultats établis dans les deux sections précédentes il résulte que la fonction $u(x, t)$ qui représente le mouvement de la corde en présence de lobstacle $u=-h$ est donnée par les formules

$$
\begin{gathered}
u(x, t)=i\left\{\frac{F(x+t)+F(x-t)}{2}, \frac{F(x+t)-F(x-t)}{2}\right\} \\
F(y)=\frac{u_{0}(y)-1}{2} \text { pour }-\frac{1}{2} \leqslant y \leqslant a<\frac{1}{2} \\
F(y)=\frac{1-u_{0}(y)}{2} \text { pour }-\frac{1}{2}<a \leqslant y \leqslant \frac{1}{2} .
\end{gathered}
$$

Les relations (17) et (11) définissent la fonction $F$ pour tout $y$, cette fonction, nulle pour $y=a$, est bien non décroissante. La formule (16) va nous permettre, à laide des propriétés des fonctions $F$ et $\dot{v}$ d'obtenir, en ce qui concerne le mouvement de la corde. les propriétés énoncées dans les théorèmes 1,2 et 3 .

\section{Thèorème 1}

Lorsque la corde est initialement au repos dans une position $u_{0}(x)$ satisfaisant les hypothèses $(\mathscr{H})$, son mouvement en présence de l'obstacle $u=-h$ est une fonction presque-périodique du temps.

Nous remarquons d'abord que, $s i h$ est supérieur ou égal à 1, la présence de l'obstacle ne perturbe pas le mouvement de la corde qui est donc périodique de période 2 . Nous supposerons donc désormais que l'on a $0 \leqslant h<1$.

Nous rappelons qu'une fonction $f(t)$ est dite presque-périodique si à tout nombre positif $\varepsilon$ on peut faire correspondre une longueur $1(\varepsilon)$, appelée intervalle d'inclusion, telle que tout intervalle de longueur 1 contienne un nombre $\tau$ (presque-période) pour lequel on a quel que soit $t$

$$
|f(t+\tau)-(t)|<\varepsilon \text {. }
$$

Il est en général difficile de démontrer qu'une fonction est presque-périodique en prouvant l'existence des grandeurs $1(\varepsilon)$ et $\tau$. Il est plus simple d'utiliser le critère de Böchner ([10] pages 77 sq.) dont nous rappelons l'énoncé: "Pour qu'une fonction $f(t)$ de la variable réelle $t$, définie et continue pour toutes les valeurs de $t$, soit presque-périodique, il faut et il suffit que de toute suite $f\left(t+t_{n}\right)$ où les $t_{n}$ sont des nombres réels arbitraires on puisse extraire une sous-suite $f\left(t+t_{1}\right)$ qui, pour toutes les valeurs de $t$, converge uniformément vers une valeur limite."

Pour appliquer ce critère, nous remplaçons dans la formule (16) $t$ par $t+t_{n}$. Nous posons ensuite

$$
\left.\begin{array}{rl}
t_{n} & =2 p_{n}+\rho_{n} \quad p_{n} \text { entier, } 0 \leqslant \rho_{n}<2 \\
2 p_{n} & =(1+h) q_{n}+\sigma_{n} \quad q_{n} \text { entier, } 0 \leqslant \sigma_{n}<1+h .
\end{array}\right\}
$$

Si on remarque que, d'après la seconde des formules (11), on a $F\left(y \pm 2 p_{n}\right)=F(y) \pm 2 p_{n}$, et 
que, d'après la périodicité de la fonction $\bar{v}(X, T)$, on a $\tilde{v}\left(X, T+2 p_{n}\right)=\dot{v}\left(X, T+\sigma_{n}\right)$, on peut écrire

$$
u\left(x, t+t_{n}\right)=\bar{v}\left\{\frac{F\left(x+t+\rho_{n}\right)+F\left(x-t-\rho_{n}\right)}{2}, \frac{F\left(x+t-\rho_{n}\right)-F\left(x-t-\rho_{n}\right)}{2}+\sigma_{n}\right\} .
$$

Le suite $\rho_{n}$ étant bornée possède au moins un point d'accumulation $\rho$, et il existe une sous-suite $\rho_{m}$ qui tend vers $\rho$. La suite $\sigma_{m}$ étant également bornée possède à son tour au moins un point d'accumulation $\sigma$, et il existe une sous-suite $\sigma_{1}$ qui tend vers $\sigma$. La fonction $F(y)$ est uniformément continue pour toutes les valeurs de $y$; la fonction $v(X, T)$ est uniformément continue en $T$ car périodique et uniformément continue en $X$ sur tout compact. La somme $2 X=F\left(x+t+t_{1}\right)+F\left(x-t-t_{1}\right)$ est inférieure à 1 en module, car $F^{\prime}(y)$ étant positif ou nul, $2 X$ est une fonction non décroissante de $x$, qui, dans lintervalle $-1 / 2 \leqslant x \leqslant 1 / 2$, varie de -1 à 1 en vertu des relations (10). En conséquence, lorsque 1 augmente indéfiniment, la suite $U\left(x, t+t_{1}\right)$ converge uniformément vers la fonction limite

$$
u^{*}(x, t)=\tilde{v}\left\{\frac{F(x+t+\rho)+F(x-t-\rho)}{2}, \frac{F(x+t+\rho)-F(x-t-\rho)}{2}+\sigma\right\} .
$$

Ce résultat établit la presque-périodicité de la fonction $u(x, t)$ considérée comme fonction du temps. Un certain nombre de propriétés mathématiques liées à cette presque-périodicité seront démontrées dans une publication ultérieure [11].

\section{Théorème 2}

Lorsque la corde est initialement au repos dans une position $u_{0}(x)$ satisfaisant les hypothèses $(\mathscr{H})$ et lorsque $h$, compris entre 0 et 1 , est un nombre rationnel: $h=p / q$ ( $p$ et $q$ entiers), le mouvement de la corde est une fonction périodique du temps de période $p+q$ si $p+q$ est pair, de période $2(p+q)$ si $p+q$ est impair.

La fonction $F(y)-y$ est périodique de période 2 ; la formule (6) nous montre alors que, si la fonction $v(x, t)$ est périodique en $t$ avec une période $\theta$ égale à un nombre entier pair, alors la fonction $u(x, t)$ est périodique en $t$ avec la même période. La fonction $\tilde{v}(X, T)$ est périodique en $T$ avec la période $1+h=(p+q) / q$ donc avec la période $p+q$. La formule (16) indique alors que, si le nombre $p+q$ est pair, la fonction $u(x, t)$ est periodique en $t$ avec la même période. Si $p+q$ est impair, $2(p+q)$ est une période entière et paire pour $\tilde{v}(X, T)$ donc pour $u(x, t)$ considérées comme fonctions de $T$ et de $t$ respectivement.

\section{Théorème 3}

Lorsque la corde est initialement au repos dans une position symétrique $u_{0}(x)=u_{0}(-x)$ satisfaisant les hypothèses $(\mathscr{H})$ et lorsque $h$, compris entre 0 et 1 , est un nombre irrationnel, le mouvement de la corde est une fonction du temps qui n'est pas périodique saif si on a $u_{0}(x)=1-2|x|$, auquel cas le mouvement est périodique de période $1+h$.

L'intérêt de ce dernier théorème est de prouver que, parmi les mouvements de la corde en présence de l'obstacle $u=-h$, mouvements qui sont toujours presque-périodiques, il en existe certains qui, contrairement à ceux envisagés dans le théorème 2 , ne sont pas périodiques. Pour obtenir ce nouveau résultat, qui va être démontré en raisonnant par l'absurde, nous avons dû nous limiter, contrairement aux cas précédents, à une corde dont la position initiale est symétrique [fonction $u_{0}(x)$ paire].

Si le mouvement de la corde était périodique en $t$ avec la période positive $\theta$, le mouvement du milieu serait périodique avec la même période. La symétrie de la position initiale de la corde a pour conséquence, en vertu des relations (17), que la fonction $F(y)$ est une fonction impaire. Comme on a par ailleurs $\partial \bar{v} / \partial T= \pm 2$, on peut écrire successivement

$$
\left.\begin{array}{c}
u(0, t)=\tilde{v}\{0, F(t)\} \\
\frac{\partial u}{\partial t}(0, t)= \pm 2 F^{\prime}(t)
\end{array}\right\}
$$




$$
\left.\begin{array}{rl}
\frac{\hat{c} u}{\hat{c} t}(0, t+\theta) & =\frac{\hat{c} u}{\hat{c} t}(0, t) \\
F^{\prime}(t+\theta) & = \pm F^{\prime}(t) .
\end{array}\right\}
$$

Comme la fonction $F$ est non décroissante, seul le signe + est à prendre en considération dans la seconde des relations (23), ce qui prouve que $F^{\prime}(t)$ est périodique de période $\theta$. La fonction $F^{\prime}(t)$ ayant également la période 2 en vertu de la seconde des relations (11), deux cas sont possibles: ou bien $\theta$ est rationnel, ou bien $F^{\prime}(t)$ est constant.

Dans le premier cas, $\theta=p / q$ ( $p$ et $q$ entiers), $2 p$ qui est un multiple de $\theta$ est également une période; comme $F(t+2 p)=F(t)+2 p$, on a

$$
\left.\begin{array}{c}
u\{0, t+2 p\} \equiv u\{0, t\} \\
\tilde{v}\{0, F+2 p\} \equiv \bar{v}\{0, F\} .\}
\end{array}\right\}
$$

Ainsi $\tilde{v}(0, T)$ possède les deux périodes $2 p$ et $1+h$; comme $\tilde{v}(0, T)$ n'est pas constant, les périodes doivent être dans un rapport rationnel, ce qui est impossible puisque $h$ est irrationnel.

On déduit de cette impossibilité que $F^{\prime}(t)$ est constant et $F(t)=a t+b$ : comme la fonction $F$ est impaire, $b$ est nul, et, comme $F(t)$ - $t$ est périodique de période 1 [en vertu de la première des relations (11) et du caractère impair de $F]$, on a $a=1$, donc $F(y)=y$ et $u(x, t)=\bar{v}(x, t)$, ce qui démontre la seconde partie du théorème.

\section{CAS D'UN OBSTACLE PONCTUEL}

La méthode du changement de variables, exposée dans la Section 3, permet de traiter non seulement le cas du mouvement de la corde en présence d'obstacles rectilignes, mais aussi le cas du mouvement en présence d'obstacles ponctuels fixes, situés sur l'axe $x=0$, au point $u=h$.

La condition de réflexion de la corde sur l'obstacle est supprimée et doit être remplacée par une condition de contact. Au cours du mouvement, la condition (25) qui exprime que le milieu de la corde est au-dessus de l'obstacle doit être satisfaite; le contact n'est plus instantané mais a lieu aussi longtemps que la réaction $R$ de l'obstacle sur la corde demeure

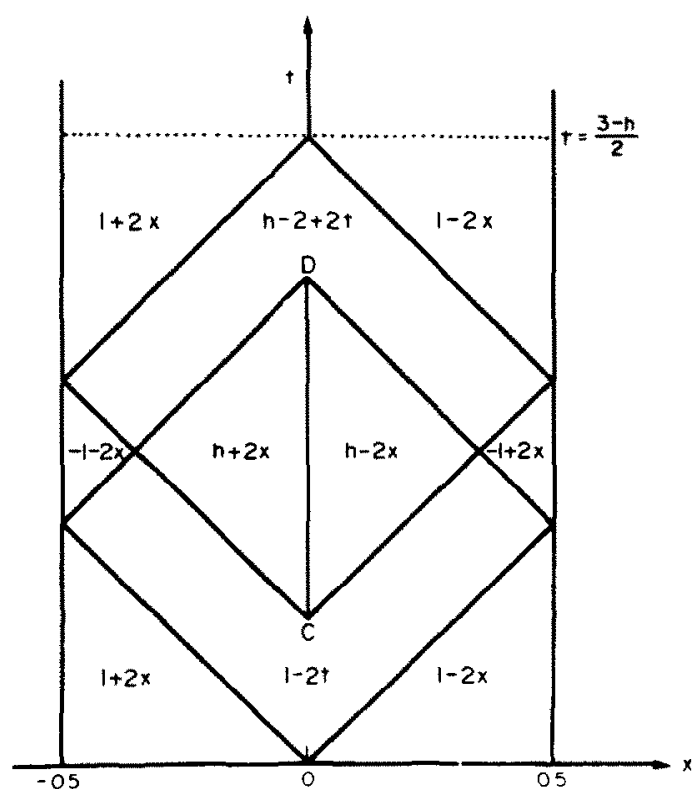

Fig. 5 Mouvement de la corde pincée symétrique. obstacle ponctuel $x=0, u=h$. 
dirigée de l'obstacle vers la corde; cette condition conduit à l'inégalité (26), cf. [5]

$$
\begin{gathered}
u(0, t)>h \\
R=\frac{\partial u}{\partial x}\left(0^{-}, t\right)-\frac{\hat{\partial} u}{\hat{c} x}\left(0^{+}, t\right) \geqslant 0 \text { pour } u(0, t)=h
\end{gathered}
$$

A l'instant initial, la corde est au repos; sa position initiale $u(x, 0)=u_{0}(x)$ est symétrique, $u_{0}(-x)=u_{0}(x)$, et satisfait les hypothèses $(\mathscr{H})$. La formule (6) permet encore dobtenir le mouvement $u(x, t)$ si on prend pour fonction $v(x, t)$ la fonction $\tilde{v}(x, t)$ qui représente le mouvement de la corde pincée symétrique et pour fonction $F(y)$ la fonction impaire définie par

$$
2 F(y)=1-u_{0}(y) \text { pour } 0 \leqslant y \leqslant \frac{1}{2}
$$

et prolongée par les conditions (11).

Le nombre $h$ étant compris entre -1 et 1 , les valeurs de la fonction $\tilde{\tilde{v}}(x, t)$ définie par les conditions initiales $\tilde{\hat{v}}(x, 0)=1-2|x|,(\hat{c} \tilde{v} / \hat{c} t)(x, 0)=0$, sont représentées sur la Fig. 5 . Le contact commence à l'instant $t=(1-h) / 2$ et se termine à l'instant $t=1$; durant le contact, segment $C D$, on a $R=4$. Le mouvement est périodique de période $\theta=(3-h) / 2$. On en déduit, comme dans la section précédente, que la fonction $u(x, t)$ est une fonction presquepériodique du temps, fonction qui n'est périodique que dans deux cas: (1) $u_{0}(x)=1-2|x|$ (2) $h$ rationnel, $h=p / q$ ( $p$ et $q$ entiers). Dans le premier cas, la période a pour valeur $\theta=(3-h) / 2$, dans le second cas $\theta=3 q-p$.

\section{CONCLUSION}

Les phénomènes physiques comportant des vibrations en présence d'obstacles sont fort nombreux. On peut citer, en génie civil, les vibrations des structures en présence d'un support en béton, en biomécanique les vibrations du tympan en présence des osselets, en acoustique les vibrations des cordes de piano en présence des marteaux. et d'une façon générale tous les phénomènes d'écho. Naturellement le modèle que nous étudions est un schema très simplifié de réalités fort complexes; même si la modélisation utilisée est dérisoire par rapport à cette complexité, on ne peut en déduire qu'elle soit mauvaise ou inutile. Les problèmes mathématiques introduits sont nombreux et intéressants, et nous espérons que notre travail pourra servir de point de départ à des travaux ultérieurs aussi bien appliqués que théoriques.

\section{BIBLIOGRA PHIE}

1. J. D'Alembert. Opuscules mathématiques. David édıteur. Paris (1761).

2. L Amerio et G. Prouse, Study of the motion of a strnng vibrating against an obstacle. Rendiconti di Matematica 8, $563(1975)$.

3 M. Schatzman. An hyperbolic problem of second order with unilateral constraints: the vibrating string with a concave obstacle. Publ. Université Paris VI. no 78031.

4. C. Reder, Etude qualitative d'un problème hyperbolique avec contrainte unilatérale Thèse de lème cycle. Université de Bordeaux (1979).

5. H. Cabannes, Mouvements périodiques d'une corde vibrante en présence d'un obstacle ponctuel. J. Mécanique 20. $41(1981)$.

6 B. Betro et L. Gotusso. Calcolo numerico di una corda vibrante contra un obstacolo. Instituto per le applicazioni del calcolo "Mauro Picone"- Publ. serie III. no 114 (1977)

7 C. Citrini et B. d'Acunto. Sur le choc de deux cordes C.r Acad. Sci. Paris 289A. 5 (1979)

8. H. Cabannes, Mouvements périodiques d'une corde vibrante en présence d'un obstacle rectiligne. $Z$. Angew: Math. Phys. 31, 473 (1980).

9. H. Cabannes et A. Haraux. Mouvements presque-pérıodiques d une corde vibrante en présence d un obstacle rectiligne. C. r. Acad. Sci. Paris 291A. 563 (1980)

10. J. Favard. Lesons sur les fonctions presque-periodqques Gauthier-Villars. Parıs (1935)

11. A Haraux et $\mathbf{H}$. Cabannes. Almost periodic motion of a strıng vibrating against a straight fixed obstacle. $J$. Nonlinear Anal, à paraitre. 


\section{Résumé:}

On conşidère les mouvements plans d'une corde vibrante, fixée à ses extrémités, en présence d'un obstacle fixe, rectiligne, parallèle à la position d'équilibre de la corde. La corde rebondit sur l'obstacle suivant les lois de la réflexion parfaite. On démontre que, sous des conditions assez générales, le mouvement de la corde, initialement au repos, est une fonction presque-périodique du temps. Lorsqu' un certain rapport est rationnel, le mouvement est périodique, et on calcule la période. On prouve ensuite l'existence de mouvements presque-périodiques mais non périodiques. Dans une dernière partie, on considère le cas d'un obstacle ponctuel fixe, placé sur la médiatrice du segment qui joint les extrémites fixes de la corde; on obtient des résultats analogues.

\section{Zusarmenfassung:}

Wir behandeln die ebene Bewegung einer schwingenden Saite, die an beiden Enden festgehalten ist, unter der Anwesenheit eines geraden festen Hindernisses, das parallel zur Gleichgewichtslage der Saite ausgerichtet ist. Der Rückprall der Saite von dem Hindernis folgt den Gesetzen der idealen Reflektion. Wir zeigen, dass unter ziemlich allgemeinen Bedingungen die Bewegung der ursprünglich ruhenden Saite eine fast periodische Funktion der zeit ist. Wenn ein bestimmter parameter sich als rationale Zahl erweist ist die Bewegung periodisch und wir berechnen die Periode; wir beweisen dann das Vorhandensein fast periodischer jedoch nichtperiodischer Bewegungen. Im letzten Teil betrachten wir den Fall eines Punkthindernisses mit fester Masse, welches auf der normalen Mittellinie des Saitensegmentes zwischen den festen Enden der Saite liegt; wir erhalten ähn Tiche Ergebnisse. 